Documentation et bibliothèques

DOCUMENTATION BIBLIOTHEQUES

\title{
Pour un système national d'information québécois
}

\section{Jean-Rémi Brault}

Volume 25, numéro 3, septembre 1979

URI : https://id.erudit.org/iderudit/1054320ar

DOI : https://doi.org/10.7202/1054320ar

Aller au sommaire du numéro

\section{Éditeur(s)}

Association pour l'avancement des sciences et des techniques de la documentation (ASTED)

ISSN

0315-2340 (imprimé)

2291-8949 (numérique)

Découvrir la revue

Citer cet article

Brault, J.-R. (1979). Pour un système national d'information québécois. Documentation et bibliothèques, 25(3), 125-131.

https://doi.org/10.7202/1054320ar

\section{Résumé de l'article}

Depuis la Conférence intergouvemementale sur la planification des infrastructures en matière de documentation, de bibliothèques et d'archives, tenue en 1974, l'Unesco a publié plusieurs documents importants qui incitent les gouvernements à doter leur pays d'un système national d'information et décrivent la méthodologie de l'implantation d'un tel réseau national. L'auteur propose une adaptation et une application de ces documents au contexte québécois, en tenant particulièrement compte d'un certain nombre de documents publiés par le gouvernement québécois, documents qui permettent d'en espérer la réalisation.
Tous droits réservés $@$ Association pour l'avancement des sciences et des techniques de la documentation (ASTED), 1979
Ce document est protégé par la loi sur le droit d'auteur. L’utilisation des services d'Érudit (y compris la reproduction) est assujettie à sa politique d'utilisation que vous pouvez consulter en ligne.

https://apropos.erudit.org/fr/usagers/politique-dutilisation/ 


\title{
Pour un système national d'information québécois
}

\author{
Jean-Rémi Brault \\ Conservateur en chef \\ Bibliothèque nationale du Québec \\ Montréal
}

Depuis la Conférence intergouvernementale sur la planification des infrastructures en matière de documentation, de bibliothèques et d'archives, tenue en 1974, I'Unesco a publié plusieurs documents importants qui incitent les gouvernements à doter leur pays d'un système national d'information et décrivent la méthodologie de l'implantation d'un tel réseau national. L'auteur propose une adaptation et une application de ces documents au contexte québécois, en tenant particulièrement compte d'un certain nombre de documents publiés par le gouvernement québécois, documents qui permettent d'en espérer la réalisation.

Since the intergovernmental conference on the plannification of the infrastructures in the fields of documentation, libraries and archives, held in 1974, Unesco has published several important documents urging the governments to equip their country with a national information system, and describing the methodology for the implantation of such a national network. The author proposes an adaptation and an application of these documents to the Quebec context, while taking into account a certain number of documents published by the Quebec government, documents which allow hope for its realization.

Desde la Conferencia intergubernamental sobre la planificación de las infraestructuras en materia de documentación, bibliotecas y archivo, que tuvo lugar en al año 1974, la UNESCO publicó varios documentos importantes que incitan los gobiernos a establecer en su país un sistema nacional de información y que describen la metodología necesaria para implantar una red nacional de este tipo. El autor propone una adaptactión y aplicación de estos documentos al contexto quebecense, teniendo cuenta particularmente de algunos documentos publicados por el gobierno de Quebec que permiten creer en la realización de tal proyecto.

L'établissement d'un organisme de coordination des réseaux de bibliothèques québécoises constitue un projet important. Compte tenu des nombreuses implications du projet, dont la création d'un véritable système d'information n'est pas la moindre, il m'apparaît que ce projet revêt pour ce secteur autant d'importance qu'on en a accordé il y a quelques années au code des professions ou à l'assurance-automobile dans leur secteur respectif. L'incohérence qui règne dans le domaine de la documentation et de l'information, le gaspillage qui s'y infiltre et dégénère en aliénation financière et culturelle, exigent une décision ferme et contraignante. En même temps que le projet vise à assurer la coordination des bibliothèques et des centres de documentation et d'information, il veut aussi donner au Québec un contrôle réel sur les sources intellectuelles et techniques de l'information et respecter le droit de tous les citoyens à l'information. Ce projet s'inscrit carrément dans le proces- 
sus de libération amorcé face à divers types de dépendances économiques, intellectuelles, technologiques ou autres. Déjà, les résultats de la réunion du Lac Beauport et les travaux plus récents de la Table ronde ${ }^{1}$ ont démontré la volonté ferme des spécialistes québécois de la documentation d'obtenir cet instrument de planification.

Dans le présent texte, j'ai l'intention d'expliquer dans quel cadre législatif un tel projet peut se réaliser. En m'inspirant des documents produits par l'Unesco, je délimiterai le contenu de la législation dont le Québec a besoin pour réaliser ce projet.

\section{NATIS}

Rappelons d'abord que le concept de NATIS ${ }^{2}$ inclut l'organisation des systèmes nationaux d'information. II a été adopté lors de la Conférence intergouvernementale sur la planification des infrastructures en matière de documentation, de bibliothèques et d'archives, organisée par l'Unesco et tenue à Paris du 23 au 27 septembre 1974, avec la collaboration de la Fédération internationale de documentation (FID), de la Fédération internationale des associations de bibliothécaires et des bibliothèques (IFLA) et du Conseil international des archives (C|A).

En adoptant le principe du NATIS, les participants à cette Conférence proposaient aussi les douze "objectifs à assigner à ces systèmes qui engloberaient l'ensemble des services concourant à assurer l'information de tous les secteurs de la communauté et de toutes les catégories d'utilisateurs. ${ }^{3}$

A la suite de cette Conférence et de l'adoption de ses recommandations par la Conférence générale de I'Unesco, «un

1. Voir à ce sujet l'article de Réal Bosa, «La planification nationale des services d'information documentaire», Documentation et bibliothèques, vol. 24, no 3 (septembre 1978), 129-135.

2. Le sigle NATIS signifie: National Information System.

3. Philipp Sewell, A.W. Mabbs et E.M. Broome, Institution d'un cadre législatif pour la mise en place du NATIS, Paris, Unesco, 1977, p. 3. groupe de travail ad hoc du NATIS (...) a été constitué pour servir de mécanisme permanent de consultation, pour conseiller le Secrétariat à l'occasion de la préparation des directives et pour assurer un contact permanent avec les spécialistes de la documentation, des bibliothèques et des archives".

C'est dans le contexte de ces travaux qu'ont été préparées et publiées les trois premières directives. La première s'adresse aux personnes qui ont la responsabilité des décisions et s'intitule La politique nationale d'information ${ }^{4}$. La deuxième s'adresse aux autorités gouvernementales qui veulent créer un NATIS et s'intitule Conception et planification des systèmes nationaux d'information: étude à l'usage des responsables gouvernementaux de la planification ${ }^{5}$. Enfin, la troisième directive "passe en revue les questions qui devraient faire l'objet de dispositions dans les textes législatifs." Elle s'intitule Institution d'un cadre législatif pour la mise en place du NATIS ${ }^{7}$.

\section{Établissement d'un NATIS québécois}

A la lumière des travaux déjà exécutés au Québec, dans le contexte des documents déjà produits par l'Unesco, je suggère que le NATIS québécois pourrait être établi en respectant les étapes suivantes.

1. Il faut d'abord que le gouvernement se laisse convaincre de l'impérieuse nécessité de se doter d'un système national d'information et, par voie de conséquence, de légiférer dans ce sens. L'Unesco rappelle que «de plus en plus conscients de ce que l'information est non seulement une ressource nationale indispensable au progrès scientifique et économique, mais aussi un agent de communication

4. D.J. Urquhart, La politique nationale d'information, Paris, Unesco, 1976.

5. Björn Tell, Conception et planification des systèmes nationaux d'information: étude à l'usage des responsables gouvernementaux de la planification, Paris, Unesco, 1976.

6. Philipp Sewell et al., Institution d'un cadre..., p. 4. 7. Ibid. 
sociale, de nombreux gouvernements sont conduits à planifier systématiquement leurs systèmes nationaux d'information pour pouvoir, en coordonnant les divers éléments constructifs, utiliser au mieux les ressources et les moyens disponibles en matière d'information. " 8

C'est donc à cette prise de conscience nationale qu'il faut inviter les autorités gouvernementales. Et cette prise de conscience doit déboucher sur une ferme volonté politique qui, elle-même, doit se traduire par une législation.

Sans verser dans un chauvinisme narcissique, il convient de rappeler que, faute de cette coordination, le Québec s'est installé dans un réseau de dépendance face aux instruments que se sont donnés nos voisins américains et canadiens. Cette dépendance, qui n'est pas loin de devenir une aliénation culturelle, se traduit également par l'exportation de ressources financières qui pourraient être avantageusement utilisées dans la mise sur pied d'un réseau national.

Non seulement sommes-nous dépendants, mais les réseaux d'information documentaire québécois stagnent dans une magnifique incohérence. Des centaines de centres documentaires sont dispersés à travers le Québec, se développent souvent d'une façon inflationniste et, surtout, isolée. Et, paradoxalement, pour une multitude de raisons, les citoyens continuent d'être privés d'un accès commode à l'information documentaire dont ils ont un besoin légitime.

Déjà, le gouvernement du Québec a laissé entrevoir des signes non équivoques de cette prise de conscience nécessaire et préalable à toute action législative. N'affirme-t-il pas dans le document Pour une politique québécoise de la recherche scientifique que "l'information scientifique et technique est de plus en plus considérée comme une ressource de premier ordre, tout

8. Ibid., p. 5. aussi indispensable pour le développement économique, social et culturel que pour le développement scientifique lui-même. ${ }^{9}$

Aussi, il m'apparaît que la problématique a été fort bien et maintes fois posée. Toutes les facettes du problème ont été scrutées et analysées. Tous les éléments du dossier sont disponibles pour prendre la décision qui s'impose depuis longtemps: je pense au dossier réuni pour la Table ronde sur la planification des services documentaires québécois ${ }^{10}$, je pense aux nombreux rapports depuis CID$B E Q^{11}$ jusqu'à CERBIQ ${ }^{12}$, je pense au livre vert Pour une politique québécoise de la recherche scientifique et au livre blanc sur La politique québécoise du développement culture/ ${ }^{13}$. Peu de pays, sans doute, ont pu élaborer une législation à partir d'un constat aussi élaboré et aussi copieux. II faut souhaiter que la multiplicité des analyses et la variété des verdicts ne dissimulent pas les véritables solutions, à la manière des arbres dont on dit volontiers qu'ils peuvent cacher la forêt.

II reste à prendre la décision politique de doter ce pays d'une législation adéquate pour, à l'instar d'un grand nombre d'autres pays, doter celui-ci

9. Québec, Ministère d'État au Développement culturel, Pour une politique québécoise de la recherche scientifique, Québec, Éditeur officiel, 1979, p. 194.

10. Les principaux documents relatifs à cette Table ronde seront prochainement publiés par l'ASTED.

11. Je rappelle pour ceux qui l'ignoreraient ou qui l'auraient oublié que, dès 1974, CIDBEQ (Centre d'informatique documentaire des bibliothèques d'enseignement du Québec) était un projet coopératif de catalogage automatisé du Service général des moyens d'enseignement du ministère de l'Éducation. Au moins dans un premier temps, il s'adressait surtout aux bibliothèques des collèges.

12. Québec, Ministère des Communications, Service de la documentation, CERBIQ: vers un réseau québécois des bibliothèques. Rapport du Comité d'étude d'un réseau des bibliothèques québécoises pour le traitement en coopération de leur documentation, Québec, 1978, 175 p.

13. Québec, Ministère d'État au Développement culturel, La politique québécoise du développement culturel, Québec, Éditeur officiel, 1978, 2 vol., $472 \mathrm{p}$. 
d'un système national d'information. «ll faut vouloir les moyens tout autant que la fin, et, au besoin, adapter la fin aux moyens. " 14

2. La législation à créer devra revêtir un certain nombre de caractéristiques, parmi lesquelles il convient d'identifier les suivantes.

2.1 La législation devra prévoir des mécanismes de coordination et non de simples préoccupations de planification. "Alors que la planification peut s'effectuer en l'absence de dispositions législatives particulières, il faudra en règle générale adopter une législation pour que la coordination ait un caractère plus systématique que celle qui peut procéder d'un accord spontané." ${ }^{15}$ Cette coordination est exigée tant par la situation chaotique où se trouvent maintenant les divers réseaux de bibliothèques que par l'anarchie et l'esprit monopolisateur qui caractérisent leur développement. A partir du principe émis par l'Unesco - et sur lequel on n'insistera jamais assez - à savoir que l'information est «une ressource nationale indispensable au progrès scientifique et économique» ${ }^{16}$, à partir du désir impératif exprimé par le livre vert Pour une politique québécoise de la recherche scientifique que "la constitution de ce réseau devrait aller de pair avec la mise sur pied d'un organisme associé au développement et à la coordination des services d'I.S.T. au Québec»17, il est absolument requis que cette législation permette de coordonner le développement rationnel des réseaux québécois d'information, qu'elle donne à l'organisme qui sera créé et qui sera chargé de

14. Philipp Sewell et al., Institution d'un cadre..., p. 19.

15. Ibid., p. 29

16. Ibid., p. 5.

17. Québec, Ministère d'État au Développement culturel, Pour une politique québécoise de la recherche..., p. 196. coordonner les moyens efficaces et contraignants pour atteindre ses objectifs.

2.2 Cette législation devra intégrer tous les réseaux existants, aussi bien ceux des bibliothèques d'enseignement pour tous les niveaux que les bibliothèques publiques et les B.C.P., les bibliothèques gouvernementales et les centres de documentation et d'information des services de santé. A cette fin, comme le suggère l'Unesco, l'organisme de coordination qui sera créé «peut, par exemple, relever directement du Conseil des ministres ou d'une autre autorité gouvernementale centrale. II sera probablement étoffé par des centres d'information rattachés aux divers ministères.»18 Ce statut "supraministériel» est important si on veut lui assurer une neutralité vis-à-vis les réseaux actuels qui relèvent de ministères différents et si, par conséquent, on veut le doter d'une capacité de cristalliser et de polariser les énergies autour d'objectifs admis par tous.

2.3 Cette législation devra prévoir un rôle important pour la Bibliothèque nationale. Maints pays qui se sont dotés d'une semblable législation ont réservé un rôle précis pour leur bibliothèque nationale. Que ce soit en Belgique où on a rattaché à la Bibliothèque royale Albert $1^{\mathrm{er}}$ le Centre national de documentation scientifique et technique; que ce soit en Grande-Bretagne où la British Library «joue une action directrice encore plus nette en ce sens qu'elle est habilitée à aider financièrement d'autres organismes (...) et qu'elle comprend un important département patronnant des travaux de recherche et de développement»19; que ce soit en Norvège où on a joint à la $\mathrm{Bi}$ bliothèque nationale «une institu-

18. Philipp Sewell et al., Institution d'un cadre..., p. 29.

19. Ibid., p. 30. 
tion pour la recherche et les bibliothèques spécialisées" ${ }^{19}$; que ce soit aux États-Unis où, même si "le système est moins structuré", la Library of Congress «assure des services centralisés et exerce une certaine autorité du point de vue technique»19; partout où on a créé une Commission nationale de coordination, on a aussi assuré à la bibliothèque nationale un rôle important et relativement précis. Or, il convient de rappeler que si, dans l'ensemble, le rôle des diverses bibliothèques est le même, s'il est fidèle aux recommandations de l'Unesco, il peut différer légèrement, selon que chacune met l'accent sur la conservation ou sur le leadership national. Or, il apparaît certain que la Bibliothèque nationale du Québec ne peut se limiter à un rôle statique et n'exercer d'autres activités que l'acquisition et la conservation du patrimoine documentaire québécois. Compte tenu de la position du Québec dans le contexte socioculturel et politique du Canada et de l'Amérique du Nord, il est requis que cette institution nationale dépasse cette dimension qu'on pourrait qualifier de passive. D'ailleurs, déjà le livre blanc sur La politique québécoise du développement cul'urel, après avoir affirmé de fa̧̧on péremptoire qu'il "devrait $\epsilon$ xister au Québec, comme dal $s$ d'autres pays, une coordination générale» (des réseaux de bibliothèques), ajoute: «La Bibliothèque nationale sera appelée à jouer un rôle important ${ }^{20}$ dans le contexte de cette coordination.

2.4 Cette législation devra porter une attention toute particulière au développement des bibliothèques publiques. Et je n'hésite pas à reproduire intégralement les principales spécifications de l'Unesco sur ce sujet:

20. Québec, Ministère d'État au Développement culturel, La politique québécoise du développement..., vol. 2, p. 380 . "les réseaux de bibliothèques publiques sont un élément essentiel du NATIS et doivent être au premier plan des préoccupations lorsque l'on envisage d'élaborer une législation;

pour se développer, les réseaux de bibliothèques doivent reposer sur une législation;

la législation doit rendre impérative la fourniture de services de bibliothèque, mais elle doit pouvoir être appliquée de façon souple et progressive;

il faut vouloir les moyens autant que la fin;

il doit exister un organisme central national ayant des responsabilités et des pouvoirs clairement définis;

la nature de cet organisme sera variable, mais elle représentera plus ou moins un équilibre entre les deux principales formes de structure institutionnelle: centralisée et décentralisée;

les services de bibliothèque devront être dotés d'organes directeurs et/ou consultatifs qui veilleront à ce que les services soient soumis à un contrôle démocratique et répondent aux intérêts des utilisateurs. ${ }^{21}$

Aussi, le souci des autorités gouvernementales québécoises de doter le Québec d'un authentique réseau de bibliothèques publiques et, pour répéter un euphémisme tant de fois utilisé, de rattraper le grave retard accumulé, ce souci recevra une impulsion dynamique. Ce développement sera désormais encadré et appuyé d'un tout cohérent. II reposera sur l'énoncé no I qui figure dans le rapport de la Conférence intergouvernementale sur le NATIS de 1974: "L'information est une part essentielle des

21. Philipp Sewell et al., Institution d'un cadre..., p. 46 . 
ressources d'une nation et l'accès à cette information constitue l'un des droits fondamentaux de l'homme».

Car, tel est bien le seul souci fondamental et essentiel qui doit présider à la création des bibliothèques publiques comme à celle d'un NATIS: assurer un légitime accès à l'information ${ }^{22}$. Et il faut insister, comme l'Unesco le fait: c'est un droit fondamental de l'homme.

La mise sur pied d'un NATIS québécois apparaît donc comme un moyen privilégié d'accélérer l'implantation d'un réseau de bibliothèques publiques.

2.5 La législation devra tenir compte, d'une façon particulière, des réseaux de bibliothèques d'enseignement, aussi bien aux niveaux élémentaire et secondaire qu'aux niveaux collégial et universitaire. Ces réseaux constituent sûrement les mieux développés; ils ont organisé les meilleures collections, ils offrent les meilleurs services, grâce à un personnel nombreux et efficace, à des locaux souvent spacieux, à des budgets souvent fort convenables.

II importe que ces réseaux continuent de se développer, maintiennent le rythme de croisière dans certains cas, rattrapant certains retards dans d'autres cas. Mais surtout, il faudra faire en sorte que chaque réseau puisse atteindre ses objectifs propres dans le respect des objectifs collectifs.

"Comme il est reconnu que, pour être efficaces, les services d'information doivent s'appuyer sur des réseaux de bibliothèques, et que les pressions économiques incitent à tirer parti au maximum de toutes les ressources disponibles, les bibliothèques universitaires doivent désormais être considérées comme d'importants éléments des systèmes nationaux d'information.» ${ }^{23}$ Or, il se trouve que les bibliothèques universitaires québécoises forment un ensemble dynamique et relativement bien pourvu. "Cet ensemble est soutenu par une enveloppe budgétaire annuelle d'environ 40 millions de dollars et par un personnel d'environ 1600 employés dont les deux tiers possèdent une formation professionnelle ou technique. Avec les bibliothèques gouvernementales et paragouvernementales et celles des entreprises privées, il forme l'élément principal d'un réseau de bibliothèques de recherche.» ${ }^{24}$

Par son poids, par l'accumulation des documents et des compétences, ce réseau peut influencer puissamment l'ensemble du système national d'information. En plus de l'intégrer, le NATIS doit s'en servir comme d'un levier, sinon d'un levain.

Des remarques semblables pourraient être faites au sujet du réseau des bibliothèques de collège. Celles-ci profitent d'une longue expérience de collaboration. Elles sont remarquables par leur dynamisme, la richesse de leurs collections, la qualité de leurs locaux et leur personnel. Aussi faut-il s'assurer que, tout en continuant d'offrir leurs services à l'institution dont elles dépendent, elles s'intègrent dans un réseau national qui profitera de leur compétence et dont elles tireront aussi des bienfaits.
22. Voir à ce sujet le Manifeste de l'Unesco sur la bibliothèque publique, révisé en 1972, ainsi que: F.M. Gardner, Législation relative aux bibliothèques publiques: étude comparative, Paris, Unesco, 1971.
23. Philipp Sewell et al., Institution d'un cadre..., p. 33.

24. Québec, Ministère de l'Éducation, Direction générale de l'enseignement supérieur, Comité technique d'évaluation des bibliothèques universitaires, Rapport, Québec, 1979, vol. 1, p. 60-61. 


\section{Conclusion}

Malgré des lacunes indéniables, bien identifiées et reconnues par les autorités gouvernementales, il reste que le Québec investit annuellement des sommes considérables pour le développement des bibliothèques. Or, faute de cohérence, ces investissements ne rapportent pas les résultats escomptés.

Bien plus, face aux besoins d'information, le Québec se comporte comme une colonie. II ne répond pas à ses besoins. II importe et produit peu. Et pourtant, le Québec possède l'infrastructure technique et l'expertise requise.

La Commission nationale posait un diagnostic semblable:

"The growth of libraries in the United States has been fragmentary and uneven. They evolved independently and do not presently constitute an orderly national system."25

Et c'est afin de remédier à cette situation que la Commission a été créée.

Semblablement, nous croyons que le Québec doit se doter, dans les meilleurs délais, d'un instrument de planification et de coordination qui rentabilisera les investissements. L'effort de réflexion de I'Unesco, les réalisations d'un grand nombre de pays, les nombreuses études produites au Québec au cours des récentes années, tout devrait inciter les autorités gouvernementales à passer à l'action.

25. U.S. National Commission on Libraries and Information Science, Toward a National Program for Library and Information Services: Goals for Action, Washington, D.C., 1975, p. 23. 